\title{
Tesis de economía 1986
}

1. "Nacionalización del comerclo exterlor del café y de la cafía de azúcar: un Intento de evaluaclón e interpreteclón".

Carmen A. Hernández Alvarenga y Evelyn E. Barraza Bonilla. $30 / 08 / 86$.

2. "Anállsis coyuntural del sector manufacturero de El Salvador 1980-1985. Mediana y grande empresa. Rama: maquinarla".

Julio César Ramlrez, José Antonio Escalante, Oscar Armando Guevara y José Manuel Huezo Flores. $22 / 03 / 86$.

3. "La reforma agrarla comó mocanismo de redlstribuclón". Guillermo Pérez Rivas y Naim Chávez Castro. 22/03/86.

4. "La Incidencia de la guerra sobre la estruclura económlca y soclal de El Salvador".

Patricia E. Santamaria Betancourt, Xenia Osegueda Garcla y Gustavo A. Quiñónez Hemández. 15/03/86.

5. "El crédito al secior Informal".

Angel Rigoberto de Paz, Nelson Humberto López Jiménez y Julia Evelyn Martínez Barraza. 05/03/86.

6. "Impuestos especificos al consumo en el proceso de reconstrucclón nacional".

Coralia Patricia Guerra Andreu, Alirio Alfonso Hernández y Humberto Cornejo Avalos. 25/01/86.

7. "Condlclones de vida de las capas medianas".

María de los Angeles Corea de Silva, Dinora Marisol Rodriguez Alemán y Alma Evelyn Villatoro Mena.

03/02/86. 
B. "Sector Informal, caso comunldad Santa Ana".

German Gonzalo Alvarado Bonilla y Elmer Arturo Amaya Quintanilla.

$17 / 02 / 86$.

9. "Causas del delerloro del sector externo de El Salvador y la pérdlde del valor del colón. 1978-1985".

Victoria Eugenia Quintanilla Vega, Francisco Angel Sorto Rivas y José Toribio Villatoro Villatoro.

$27 / 09 / 86$.

10. "El nuevo modelo de desarrollo y la recomposiclón de la clase dominente en El Salvador".

Mario Aristides Aquino Palacios.

03/10/86.

11. "La concentraclón y centralización del capltal en la Industrla manufacturera". Alma Donata Galo Bonilla de Peña. 15/03/86.

12. "El sector Informal y la satlsfacclón de las necesldades béslcas". Sandra Lilian Martinez López y Ana Marla Elena del Carmen Molina Roque. 23/01/86.

13. "Anállsle y perspectlvae de la naclonallzaclón de la bence on El Salvador". Eugenia Suay Selinger y Silvia Lorena Rubio Galeas 23/06/86.

14. "La polítlca reglonal de los Estados UnIdos y ou Incldencla hacla El Salvador". José Roberto Salguero Martínez, Rogelio Antonio Cuéllar Santos y Rafael Victor M. Ayala Morazán. 12/04/86.

15. "Caracteristlcas del mercado Informal de trabate" Gertrudis Rivera Arévalo, Sonia I. Sánchez Cuéllar. $18 / 04 / 86$.

16. "La Politica exterior norteamerlcana y su Incidencla en la economía de EJ Salvador perlodo 1980-1984: la ayuda económlca"

Rigoberto Hernández Ramos, Edgard R. Toblas Medrano y Martín Alberto Rivera Gómez.

16/05/86.

17. "Estudlo comparallvo de cooperatlvas agricolas sector reformado y tradiclonal". Miguel Wilfredo Osorio y Manuel A. Román Morales.

18. "Efectos de la crisis y el conflicto sobre los grupos urbanos de pobreza 19791985".

Lisandro Esteban Abrego Cardoza, Dagoberto Rivera Rivera y Roxana Estela Torres Sosa.

31/05/86.

19. "Estructura de la rama Industrial mecánlca en El Salvador".

Gian Franco Innocenti Rossi y Carlos Antonio Mejla Torres.

05/07/86.

20. "El dóficit fiscal y au financlamlento en El Salvador, período 1960-1985". Oscar Edmundo Anaya Sánchez y Rina Patricia Hernández.

27/06/86. 
21. "Los proyectos reformlstas en El Salvador en la década de los ochenta". Rałael Antonio Lemus Gómez y Alexander Emesto Segovia Cáceres. 02/05/86.

22. "Incldencla de la guerra en las condiclones de ylda de los propletarios y/o dependientes de llendas Informales ublcadas on el área urbana de San Salvador, durante el perlodo 1984-1985".

Ana Margarita Cuéllar Zepeda, Guadalupe Eugenia Mira Sisniega y Silvia del Carmen Navarro de Palomeke. 23/05/86.

23. "Estructuraclón y funclonamlento del transporte colectlvo de pasajeros on el érea metropolitana de San Salvador e Interdepartamental en el perlodo 19781985".

Silvia América Gonzáles de Castillo y Luz Elena Renderos Barahona. 27/06/86.

24. "Algunos elementos de la polltlca económica en El Salvador 1979-1985". Yolanda del Carmen Dimas Linqui, Ana Patricia Duarte Schlageter y Elena Rosa Larreynaga Cruz. 07/06/86.

25. "Anállsles socloeconómico del Impuesto sobre la renta de personas naturales". Miriam Lidia Zamora Rivas. 06/09/86.

\section{7}

1. "Análisls global de la matriz Insumo-producto e Identificación del modelo oconómlco salvadorefio".

Samuel Antonio Alvarado Pineda.

17/01/87.

2. "Artlculeclón agrlcultura Industrla dentro del marco del desarrollo del capltallsmo en El Salvador, visto a través de la matrlz Insumo-produclo 1978". Marla Eugenia Alvarez de Girón, José Bueno Alferez y Marcos Gregorio Sánchez Trejo 17/07/87.

3. "Artlculación Industrla serviclos". Ana Isabel Lino de Hernández y Elizabeth Marlene Tejada Arias. 31/01/87.

4. "La evolución de la deuda externa y el creclmlento económlco en El Salvador. Periodo 1960-1985".

Jaime Renato Argueta Ruiz, José Gonzalo Montoya Alvarado y Néstor Mauricio Martinez Menéndez.

31/01/87.

5. "Necesldades béslcas y reaclivación de la economia". José Napoleón Alfaro y Alba Marla Guadalupe Orellana González. 07/05/87.

6. "Artlculaclón construcclón-Industrla-sector externo". Luis Mario Corleto Valencia y Renan Gilberto Palma Reyes. $14 / 02 / 87$. 
7. Anállale sectorlal del gasto públlco para el perlodo 1960-1985".

Hada Desirés Arteaga Rubio, Silvia Elena Chávez Molina y Ana Vilma Olmedo Recino.

08/04/87.

B. "Lae políticas de estabilizaclón y su Incidencla en El Selvador".

Ana Margoth Arévalo Cornejo, Ricardo Salvador Flores Ortiz y Luis Alonso Peralta Cerritos.

07/02/87.

9. "La Inflaclón en El Salvador período 1979-1983: un enfoque monetarlata". Ernesto Antonio Arce Molina, Sonia Velquis Portillo Escobar y Guillermo Feliciano Alvarenga.

28/03/87.

10. "Evoluclón y andillale critlco de la tributacłón en El Salvador: baees para una reforma trlbutarla".

Mario Antonio Cema Torres y Mario Antonio Martinez Rodriguez.

21/02/87.

11. "Artlculación del sector finenclero, sector agropecuarlo y eector Industrial en el marco del desarrollo del slatema capitallsta on El Salvador".

Emilia Isabel Cuéllar Velasco y Ana Gloria Mungula Martinez. 09/05/87.

12. "Funclonalldad y articulaclón de los obreros de la Industrla manufacturera on la economia salvadorefia: el caso de los obreros de la Indusirla de alimentos". Anne Susana Aguilar Campos y Ana Morena Zelaya Cornejo. $22 / 08 / 87$.

13. "Las mayorias populares: estudlo de un grupo especifico: los peones del departamento de mantenimiento de carreteras de la dirección general de caminos".

Alberto Javier Alvarenga Cardoza y Rafael Antonio López González. 15/08/87.

14. "Artlculación y funclonalldad de los pequefios productores agricolae con el sistema capltallsta salvadoreño".

Aracely Portillo Campos y Delia Lorena Reyes Bonilla. 23/09/87.

15. "Artlculación y funclonalldad des pequefio productor no caplialista de calzado on el contexto de la economia capitallata selvadorefa. Caso drea metropolltana de San Salvador".

José Guillermo Hemández Coto, Juan José Martinez Cientuegos y Roberto Rodrlguez Martinez.

05/11/87.

16. "Efectos de le reforma agrarle selvadorefia, sobre las formas de producclón y lae condlctones de vida del campesino cooperativizado de FESACORA".

Aurore Elizabeth Arévalo de Quintanilla, Francisce Concepción Barrientos de Menjlvar y Reynalda Trinidad Ramos Cruz.

05/10/87.

17. "Articulación-funclonalldad y condlclones de vida de los vendedores de la calle en la economía salvadorefa caso del primer anlilo perlferlco del área metropolltana de San Salvador".

Sentiago Ricardo Martinez Buitrago y Eduardo Antonio Urquilla Bermúdez. 28/09/87. 
18. "El sector informal y su vinculacion con otras empresas" María Mercedes Rivera de Angulo $14 / 11 / 87$

19. "Anállals económlco de la estructura producilva industrial química salvadorefia 1950-1986". José Ovidio Garcla Quintanilla y Jorge Adalberto Pasasi Hernández. 21/03/87.

20. "Estudlo de la pequeña producclón mercanill en la rama de la confecclón en el área metropolltana de San Salvador".

Armando Chávez Mayorga, Marina Mélida Morán Alemán y Gloria Alicia Peña Martinez.

09/10/87.

21. "Acumulación Industrlal y polítlca fiecal en El Salvador: 1964-1984. Un onfoque kalecklano".

Miguel Antonio Chorro Serpas y Carlos Alberto Nioreno Carmona.

31/08/87.

22. "La fijaclón de preclos en la manufactura salvadorefaa".

Lorena Marizza Contreras Gutiérrez y Mélida Dolores Pérez Castillo.

$17 / 12 / 87$.

\section{8}

1. "Mecanlsmos de trasmlalón del déflclt fiscal al desequllibrlo del sector externo. En la economla salvadorefía: periodo: 1960-1986".

José Roberto Aguilar Alvarez, Raúl Antonio Granados Montecinos y Gerardo Antonio Peraza Salazar.

25/06/88.

2. "Niveles y estructura del consumo de los seclores populares: caso especilico corladores de café".

Roberto Rafael Aquino Chávez, José Wilfredo Hemández Salguero y Gabriel Antonio Pérez Alvarado.

16/07/88.

3. "Estructura agraria salvadorefia, caracteristlcas y uso de tecnología en las unidades famillares campeslnas: perspecilvas de le blolecnologia".

Edgar Armando Cabrera Lara, Nelson Oswaldo Ramlrez Jiménez y Ricardo Noel Soriano Aguilar.

20/08/88.

4. "Desarrollo de la energla eléctrica en El Salvador: estructura, característlcas y principales problemas. Perlodo 1979-1986".

Lourdes Maricela Aguilera Martinez, Ana Hazel Escrich Cañas y Jaime Alfredo Miranda Flamenco.

18/06/88.

5. "Producllvidad y empleo en la manufaclura ealvadorefia, periodo: 1950-1986". Merla Elsa Guillén Castillo y Jesús Antonio Peña Molina.

22/07/88.

6. "Umltaciones de los paquetes lecnológlcos en el agro: el caso del rlego en el dlatrlto de Zapoiltán".

Germán Emesto Morán Figueroa y Mario Emesto Silva Arguera.

16/12/88. 
7. "Artlculación y funclonalldad de las tlendes minorltarlas". Marla del Carmen Ellas, Tito Livio Guillén Sánchez y Sandra Aracely Ramirez Pozas. 22/01/88.

B. "Productlyldad agroallmentarla". Mercedes Regina Llort Zapala y Amnando Trigueros Hemández. 23/04/88.

9. "El papel del ahorro privada Interno en la dinámica de crecimlento de la oconomia selvadorefía 1960-1985". José Luis Amaya Capellano, Federico Dlaz Trejo y Dimas Flores Valladares. $18 / 02 / 88$.

10. "Estructura organizacional y comportamlento del sistema financiero". Daysi Otilia Murcia Chicas, William Alfredo Padilla Quintanilla y Oscar Romeo Zelaya Avalos.

24/02/88.

11. "Estructura y artlculación agroindustrlal on El Salvador". Jorge Alberto Donis. 10/09/88.

12. "La estructura del desequllibrlo comerclal de EI Salvador. 1986".

Aicardo Antonio Chávez Pineda, Julio Ricardo Guzmán Ortiz y Roque Ernesto Orellana Valladares. $13 / 02 / 88$.

13. "Alcances y limiles del financlamlento al sector Informal, en las ramas de Industrla y servlclos".

Ivania Maribel Alvarez Gavidia, Ana Emilia Zoraida Morales Carranza y Sandra Celia Muñoz Romero. 09/08/88.

14. "Incldencla de las actividades informales en la determinaclón del salario minlmo en El Salvador".

Lilian Dinora Navarrete Lemus y Edith Yolanda Romero Gutiérez. $17 / 02 / 88$.

15. "Estado actual y perspecllvas de la deuda externa". Luis Alberto Landaverde Peña, Darwin López Escobar y Wiltredo Ramos Corea. 26/02/8B.

16. "Incldencla del gasto público sobre la distrlbución del Ingreso: años 1978$1985^{\circ}$.

Marton Roberto Torres, Mariza Figueroa Sandoval y Mario César Morales Quiróz. $15 / 03 / 88$

17. "Causas, evolución e Impllcaclones de la dependencla alimentería en El Salvador. Subsector granos báslcos 1960-1985".

Santos Eduardo Barahona, Francisco Arturo Comejo Olivar y Francisco Antonio Vásquez Sánchez. 25/03/88.

18. "Fomación de precios en la agricultura de alimentación".

"Paymon Guillermo Alfaro Castaneda, Fidel Enrique Majano Trejo y Ada Erlyn Méndez Narváez.

22/12/88. 


\section{9}

1. "Transporte no capltallsta de pasejeros on el área metropolltana". José Emilio Márquez H. y Rolando Antonio Moreno Rodrlguez. 20/10/89.

2. "El tranaporte de carga no capltallsta en el drea metropolitana de San Salvedor".

César Augusto Avalos Peña, Julio Heriberto Benavides Hemández y Carmen Hemández Torres.

18/02/89.

3. "Realldades y perspectlvas de la fase II de la reforma egrarla". Luis Edmundo Moreno Alvergue y Mario Reni Roldán.

11/03/89.

4. "Evasión fiscal de la mlcroempresa: cun problema para el Estado o una oportunldad para impulsar la producción microempresarlal".

Manuel Baltazar Cruz Fuentes, Julia Edith Reyes Anariba y Haydeé del Carmen Rivas Dlaz.

$08107 / 89$.

5. "Empleo salarlos y organización Industrlal: un eneayo de verlficaclón de las hlpólesis de segmentecón de los mercados de trabajo".

Edgar Alvarino Cruz Palencia, Laura Patricia Cruz Palencia y Marvin Abraham De Paz González.

15/07/89.

6. "Estructura y perspectiva de la producción de azúcar en El Salvador".

Ana Lilian Lara Cublas y Maria Gladys Lara Cubias. 29/08/89.

7. "Capecldad oclosa y reactlvación económlca en EI Salvador".

Blanca Lilian Cárcamo Aguilar, Orlando Martinez Orellana y Howard Lynn Morales Salazar.

07/07/89.

8. "Estudio de las relaciones económicas entre Europa-centroamérlca: el caso de El Salvador".

Gloria Guadalupe Carranza Mena, mercedes Lazo Flores, Celia Orbelina Pérez Reyna y Sandra Elizabeth Romero Campos. 19/08/89.

9. "La Incldencla de la lase I de la reforma agrarla y naclonallzaclón del comerclo exterlor del café en la propuesta de un modelo neoconservador ante la crlsls económlca salvadoreña".

Olga Yaneth. Mena Linares, Herbert Adán Miranda y Edgardo Antonio Ponce Calderón.

$01 / 09 / 89$.

10. "El papel del refinanclamlento bancarlo: Impacto y perspectlvas en el slstema financiero y en la economla salvadoreña, periodo 1975-1987".

Nelly Noemy Moreno Rodríguez, Alma Daysi Paredes González y Julia Margarita Umaña Gracia.

$01 / 07 / 89$. 


\section{Indice General. Año II, 1989}

\section{Editorlales}

Hubo elecciones y no se violó la Constitución ..................................................... 1

La politica en la economla de ARENA .................................................................. 139

Cambiar para mejorar: ¿qué y cómo? ..................................................................... 225

Las inversiones de la realidad y el falso realismo pragmático .................................. 355

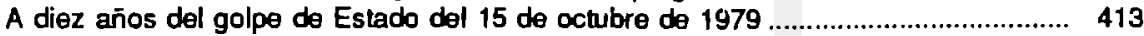

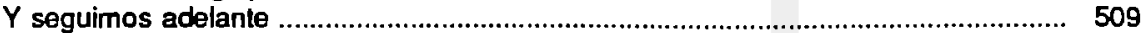

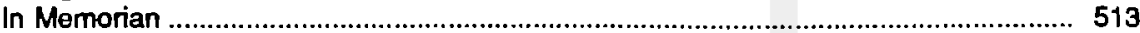

\section{Artículos}

Arrlola, Joaquin

La tecnologla adaptada: entre la realidad y el deseo .............................................. 179

Excedente potencial y desarrollo posibie ......................................................... 357

\section{Cáceres, Lula René y Suay, Roberto}

La situación entre las monedas entre El Salvador y Guatemala

\section{Goilta, Alfonso}

El Estado en momento de crisis: Redefinición del papel del Estado 1948-1960

Los efectos de las medidas del programa económico del gobiemo

\section{Hernández M., Carlos F.}

La nueva derecha en El Salvador

\section{Majano Trejo, Flded Enrlque, Méndez Narváez,}

\section{Ada Erlyn}

Formación de precios en la agricultura de alimentación

\section{Marin, José}

¿Existen pensamiento de nueva derecha en El Salvador?

\section{Montes, Segundo}

Impacto de la migración de salvadoreños a los Estados Unidos, el envio de remesas y consecuencias en la estructura familiar y el papel de la mujer

\section{Montoya, Aqulles}

La producción agrloola no capitalista y su relación con el capital

El Neo-liberalismo y la privatización de la economla 


\section{Rlvera Campos, Roberto}

Valor y distribución en Adam Smith

Producción de alimentos y desbalances macroeconómicos

Rodriguer, Tomde

Trabajo productivo y trabajo improductivo

Rosa, Herman y Sogovla, Alexander

Financiamiento extermo, deuda y transformación de la esiructura productiva de

El Salvador en la década de los ochenta: El papel de Estados Unidos.

Trlgueros A., Alvaro

Déficit fiscal $\theta$ inflación on El Salvador (1978-1987)

\section{Umaña, A.}

Cambios en la economla mundial y desallos para El Salvador

\section{Valonzuela, Ulleas}

"Evolución general de la economla salvadoreña durante 1989" 